\title{
Functional Roles of Complexin in Neurotransmitter Release at Ribbon Synapses of Mouse Retinal Bipolar Neurons
}

\author{
Thirumalini Vaithianathan, ${ }^{1}$ Diane Henry, ${ }^{1}$ Wendy Akmentin, ${ }^{1}$ and Gary Matthews ${ }^{1,2}$ \\ ${ }^{1}$ Department of Neurobiology and Behavior and ${ }^{2}$ Department of Ophthalmology, Stony Brook University, Stony Brook, New York $11794-5230$
}

\begin{abstract}
Ribbon synapses of photoreceptor cells and bipolar neurons in the retina signal graded changes in light intensity via sustained release of neurotransmitter. One molecular specialization of retinal ribbon synapses is the expression of complexin protein subtypes Cplx 3 and $\mathrm{Cplx} 4$, whereas conventional synapses express $\mathrm{Cplx1}$ and $\mathrm{Cplx} 2$. Because complexins bind to the molecular machinery for synaptic vesicle fusion (the SNARE complex) and modulate transmitter release at conventional synapses, we examined the roles of ribbon-specific complexin in regulating release at ribbon synapses of $\mathrm{ON}$ bipolar neurons from mouse retina. To interfere acutely with the interaction of native complexins with the SNARE complex, a peptide consisting of the highly conserved SNARE-binding domain of Cplx 3 was introduced via a whole-cell patch pipette placed directly on the synaptic terminal, and vesicle fusion was monitored using capacitance measurements and FM-dye destaining. The inhibitory peptide, but not control peptides, increased spontaneous synaptic vesicle fusion, partially depleted reserve synaptic vesicles, and reduced fusion triggered by opening voltage-gated calcium channels under voltage clamp, without affecting the number of synaptic vesicles associated with ribbons, as revealed by electron microscopy of recorded terminals. The results are consistent with a dual role for ribbon-specific complexin, acting as a brake on the SNARE complex to prevent spontaneous fusion in the absence of calcium influx, while at the same time facilitating release evoked by depolarization.
\end{abstract}

Key words: complexin; mouse; retina; ribbon synapse; neurotransmitter release

\section{Introduction}

The synaptic terminals of sensory neurons in the retina and inner ear release neurotransmitter tonically, and their active zones are characterized by a specialized organelle, the synaptic ribbon, which tethers large numbers of synaptic vesicles (Matthews and Fuchs, 2010). Although ribbon synapses share many molecular constituents with conventional synaptic active zones (Schmitz, 2009; Zanazzi and Matthews, 2009), an exception is complexin, which is a small, soluble protein that interacts with the molecular machinery of synaptic vesicle exocytosis, the SNARE complex (Brose, 2008; Maximov et al., 2009; Xue et al., 2010; Yang et al., 2010; Hobson et al., 2011; Martin et al., 2011). Mammals possess four complexin (Cplx) genes: conventional synapses express primarily Cplx1 and Cplx2, while retinal ribbon synapses express Cplx3 or Cplx4 (Reim et al., 2005). Mice lacking both Cplx3 and Cplx4 have visual impairment, an abnormal electroretinogram, and retinal ganglion cells that respond less well to light (Reim et al., 2009; Landgraf et al., 2012), suggesting that these Cplx subtypes are required for normal synaptic transmission in the retina.

Previously, we investigated the function of the ribbon-specific Cplx3/4 subtypes in zebrafish (Vaithianathan et al., 2013), whose

Received July 3, 2014; revised Nov. 10, 2014; accepted Dec. 10, 2014.

Author contributions: T.V. and G.M. designed research; T.V., D.H., W.A., and G.M. performed research; T.V., D.H., W.A., and G.M. analyzed data; T.V. and G.M. wrote the paper.

This work was supported by National Institutes of Health Grant R01EY003821.

The authors declare no competing financial interests.

Correspondence should be addressed to Gary Matthews, Department of Neurobiology and Behavior, Stony Brook University, Stony Brook, NY 11794-5230. E-mail: Gary.G.Matthews@stonybrook.edu.

DOI:10.1523/JNEUROSCI.2703-14.2015

Copyright $\odot 2015$ the authors $\quad 0270-6474 / 15 / 354065-06 \$ 15.00 / 0$ genome contains five genes related to mammalian Cplx3/4 (Zanazzi and Matthews, 2010). Because of the large number of Cplx3/4 isoforms that could potentially be expressed at zebrafish ribbon synapses, the previous work used an inhibitory peptide consisting of the SNARE-binding domain (SBD) of mouse Cplx3, which is expected to acutely interfere with binding of all Cplx isoforms to the SNARE complex. Actions of Cplx are somewhat variable across species and cell types (Brose, 2008) and depend on molecular context (Neher, 2010; Yang et al., 2013), making it important to investigate Cplx3/4 function at mammalian ribbon synapses as well. Therefore, in the present study, we used the inhibitory peptide to examine Cplx function in synaptic terminals of $\mathrm{ON}$ bipolar neurons from mouse retina. The results show that acutely interfering with Cplx function increased spontaneous release and inhibited evoked release in mouse bipolar neurons. We conclude that ribbon-specific complexin serves the dual functions of clamping spontaneous vesicle fusion and potentiating fusion triggered by calcium influx at mammalian ribbon synapses, much like the actions postulated for other complexins at conventional synapses.

\section{Materials and Methods}

Fluorescence imaging and patch-clamp recording. Bipolar neurons were dissociated from the retinas of adult male C57BL/6J mice after papain digestion as described previously (LoGiudice et al., 2008). For experiments using FM4-64 uptake and destaining, FM-dye uptake was stimulated by incubation in high-K saline $(80 \mathrm{~mm} \mathrm{KCl})$ plus $5 \mu \mathrm{M}$ FM4-64 (Biotium) for $90 \mathrm{~s}$, followed by washing with $1 \mathrm{~mm}$ Advasep-7 (Biotium). Cells were selected for patch-clamp recording based on intact morphology and uptake of FM4-64 restricted to synaptic terminals and were voltage clamped via a whole-cell pipette placed directly on a synaptic 
A

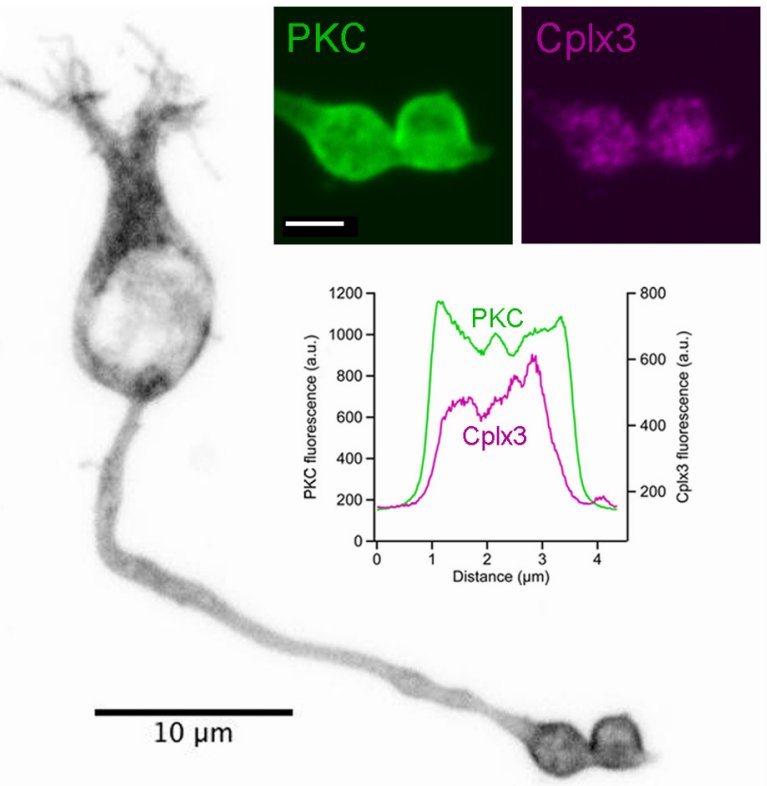

B

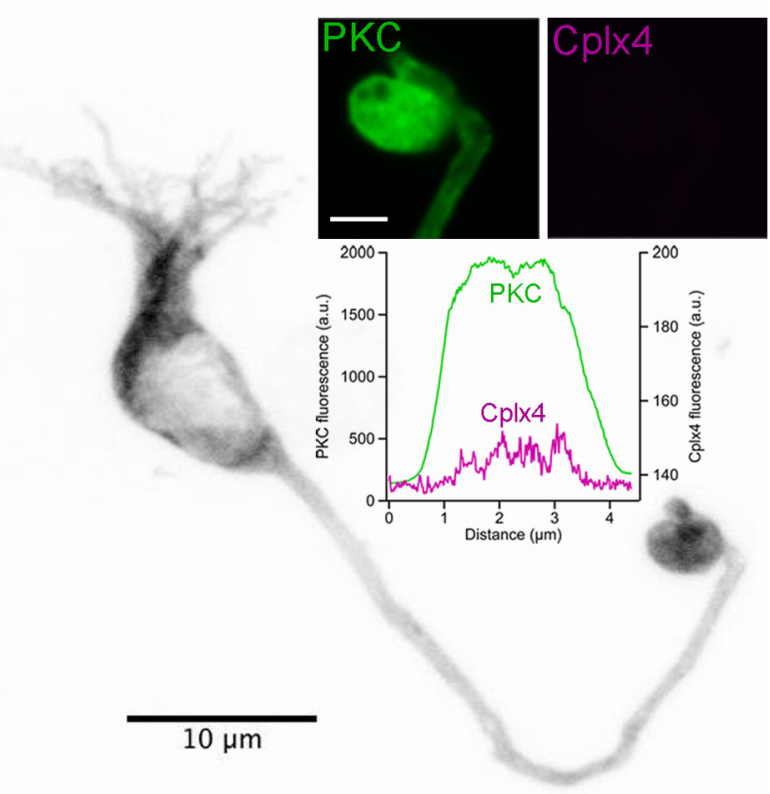

Figure 1. Immunostaining demonstrates expression of Cplx3 butnot Cplx4 in synapticterminals of mouse $0 \mathrm{~N}$ bipolar neurons. $\boldsymbol{A}, \boldsymbol{B}$, Dissociated $\mathrm{ON}$ bipolar neurons labeled with antibody against PKC $\alpha$. The intensity scale is inverted in the main panel. The upper insets show the synaptic terminal, double labeled with anti-PKC (left) and anti-Cplx3 ( $\boldsymbol{A}$, right) or anti-Cplx4 ( $\boldsymbol{B}$, right). Inset scale bars, 2 $\mu \mathrm{m}$. The lower inset shows the intensity profile across the synapticterminal for PKC (green) and (plx3 $(\boldsymbol{A}$, magenta) or Cplx4 ( $\boldsymbol{B}$, magenta). Note that the intensity scale for Cplx4 (right axis, $\boldsymbol{B}$ ) is expanded to reveal the fluorescence in the terminal, which was not significantly different from terminal staining observed with secondary antibody alone (see text).

terminal, using an EPC-9 amplifier controlled by PatchMaster software (HEKA). The pipette solution to isolate $\mathrm{Ca}^{2+}$ current was described previously (LoGiudice et al., 2008). Capacitance measurements used $1600 \mathrm{~Hz}$ sinusoidal voltage-clamp stimuli with peak-to-peak amplitude of $10 \mathrm{mV}$ centered on the holding potential $(-65 \mathrm{mV})$. Single confocal optical sections of synaptic terminals were acquired using an Olympus FV-1000/IX-81 laser-scanning confocal microscope controlled by Olympus FV10-ASW software. Photoxicity was avoided by minimizing light exposure and intensity. Images were analyzed using ImageJ (imagej.nih.gov).

Immunocytochemistry. Preparations of mouse bipolar neurons were fixed $\sim 20$ min after dissociation for $15 \mathrm{~min}$ at room temperature in $4 \%$
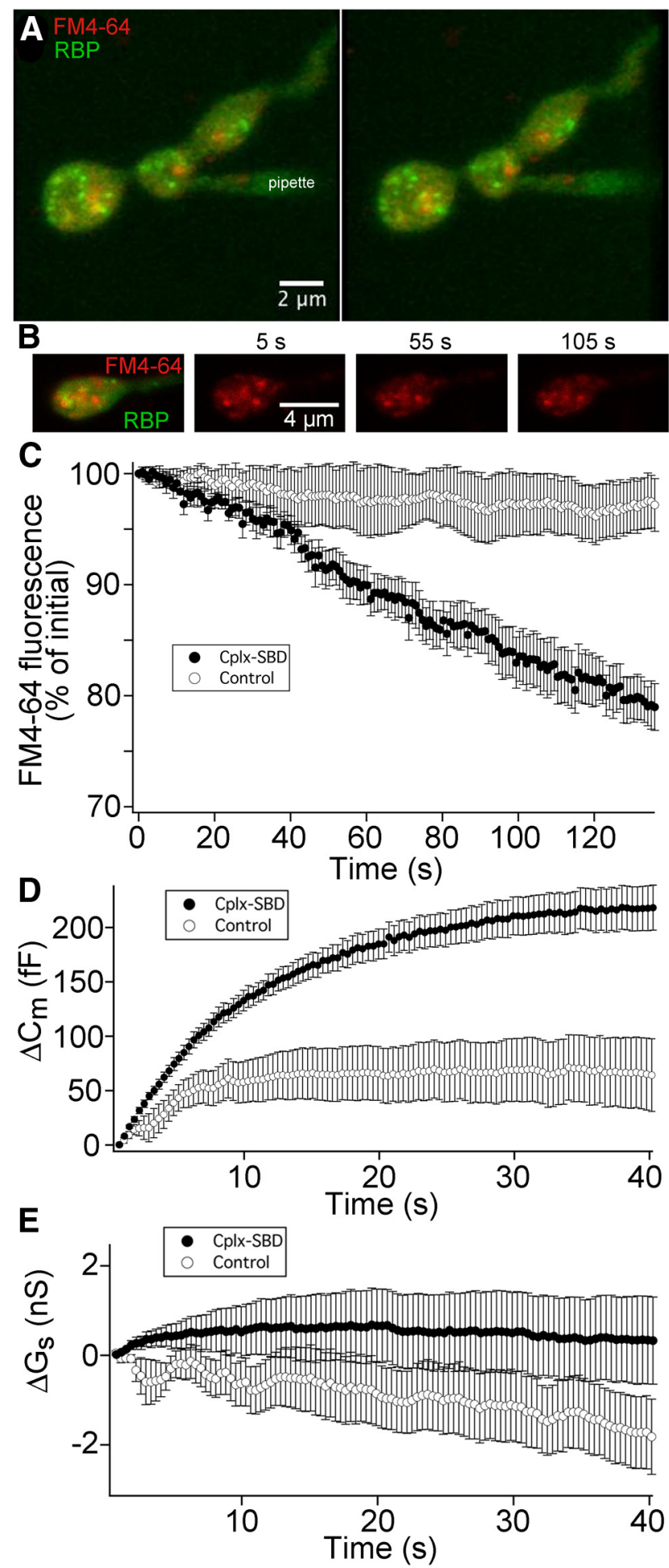

Figure 2. Peptide derived from the SNARE-binding domain (SBD) of Cplx3 induces spontaneous synaptic vesicle fusion in mouse bipolar neurons. $A$, Stereogram of synaptic terminals of an isolated mouse bipolar neuron, illustrating activity-dependent labeling of synaptic vesicles with FM4-64 dye (red). Synaptic ribbons were marked using Ribeye-binding peptide (RBP; green). Images were constructed from a $z$-axis series of confocal optical sections. B, Spontaneous loss of FM4-64 fluorescence in the synapticterminal of a mouse bipolar neuron after break-in using a whole-cell patch pipette filled with (plx-SBD. Times above images indicate time after break-in. Holding potential, -65 $\mathrm{mV}$. C, Average ( \pm SEM) FM4-64 fluorescence as a function of time after break-in for five mouse bipolar cell terminals in the presence of (plx-SBD (filled circles) and five terminals in the presence of control peptide (open circles). $\boldsymbol{D}$, Change in membrane capacitance $\left(\Delta C_{m}\right)$ as a function of time after break-in in the presence of (plx-SBD (filled circles; 11 cells) or control peptide (open circles; 11 cells). $\boldsymbol{E}$, Series conductance $\left(G_{s}\right)$ showed little change in the same recordings. 

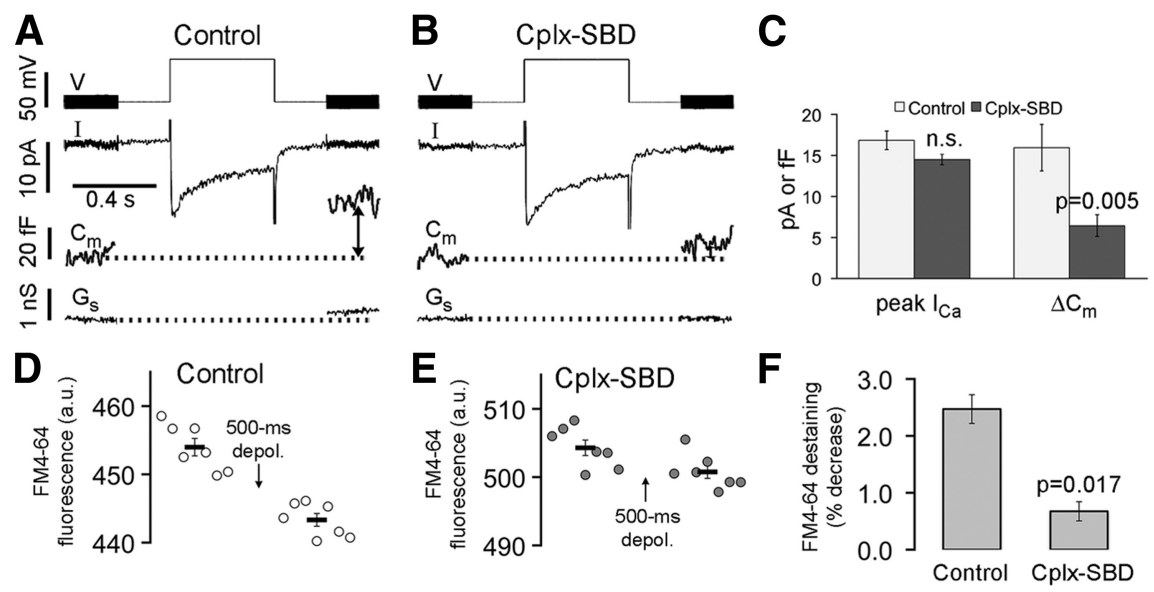

Figure 3. Cplx-SBD peptide reduced vesicle fusion evoked by depolarization. $A$, An example of calcium current $(I)$ in response to a voltage-clamp ( $V$ stimulus from -65 to $0 \mathrm{mV}$ in a cell dialyzed with control peptide. Small sinusoidal stimuli used to monitor membrane capacitance $\left(C_{\mathrm{m}}\right)$ and series conductance $\left(G_{\mathrm{s}}\right)$ are present at the beginning and end of the voltage trace. Double arrow indicates the change in $C_{\mathrm{m}}$ evoked by the stimulus. $\boldsymbol{B}$, Example of responses shown in $\boldsymbol{A}$, but in the presence of Cplx-SBD. $\boldsymbol{C}$, Comparison of calcium current (left pair) and $\Delta C_{m}$ (right pair) in 24 synaptic terminals in the presence of control peptide and 14 synaptic terminals in the presence of Cplx-SBD. D, E, Examples of the change in FM4-64 fluorescence in a mouse bipolar cell terminal elicited by a $500 \mathrm{~ms}$ voltage-clamp depolarization from -65 to $0 \mathrm{mV}$ in the presence of control peptide (D) or Cplx-SBD (E). Data points show the fluorescence intensity of the synaptic terminal in a series of seven confocal images before and seven images after depolarization. Black bars indicate the average ( \pm SEM) for each set of seven images. $\boldsymbol{F}$, Summary of evoked decrease in FM4-64 fluorescence for 29 synaptic terminals with control peptide and 19 terminals with Cplx-SBD. Error bars, \pm 1 SEM.

paraformaldehyde in PBS. Immunofluorescence staining was performed as described previously (Van Wart et al., 2007) using mouse monoclonal anticomplexin-3 (Synaptic Systems, 122311; 1:200), mouse monoclonal anticomplexin-4 (Synaptic Systems, 122411; 1:1000), rabbit polyclonal anti-PKC $\alpha$ (Santa Cruz Biotechnology, sc-10800; 1:100), Alexa 488-conjugated goat anti-rabbit secondary antibody (Invitrogen), and Cy-3-conjugated goat anti-mouse secondary antibody (Jackson Immunoresearch). To quantify immunofluorescence, the average fluorescence intensity was measured in a region of interest drawn around the synaptic terminal of a PKCpositive bipolar neuron and divided by the fluorescence intensity in the same region displaced to the side of the terminal.

Electron microscopy. In experiments to examine individual physiologically characterized bipolar neurons by electron microscopy, dissociated bipolar cells were plated onto Aclar film (Electron Microscopy Sciences). After whole-cell patch-clamp recording, the patch pipette was removed from the cell, and solution containing $2.5 \%$ paraformaldehyde and $2.5 \%$ glutaraldehyde was superfused from an application pipette. After $10 \mathrm{~min}$ of superfusion, the application pipette was removed, and the entire solution was replaced with the fixative solution. To facilitate identification of the recorded cell after embedding, the patch pipette was then used to etch a rectangle in the Aclar surrounding the recording cell. Cells were fixed overnight at $4^{\circ} \mathrm{C}$, postfixed in osmium tetroxide, and embedded and sectioned for electron microscopy (LoGiudice et al., 2008).

\section{Results}

Cplx3 but not Cplx4 is detectable by immunostaining in synaptic terminals of $\mathrm{ON}$ bipolar neurons dissociated from mouse retina

Only Cplx3 has been reported to be detectable in synaptic terminals of ON, rod bipolar neurons in mouse retina (Reim et al., 2005, 2009; Landgraf et al., 2012). For physiological experiments, described below, we used acutely dissociated ON, rod bipolar neurons, identified by their distinctive morphology. To confirm the identification of ON cells, we immunostained dissociated cells using an antibody against $\operatorname{PKC} \alpha$, which marks ON bipolar neurons. Examples of labeled bipolar neurons are illustrated in Figure 1, showing their intact morphology after dissociation. In addition, we double-labeled PKC-positive bipolar neurons with
anti-Cplx3 or anti-Cplx4, and we found strong immunostaining for Cplx3 in the terminals (Fig. 1A), as expected from the previous reports. The ratio of anti-Cplx3 fluorescence intensity in the terminal to the adjacent background intensity was $2.9 \pm 0.2($ mean $\pm \mathrm{SEM} ; N=25)$, which is significantly higher than the fluorescence observed when the Cplx antibody was omitted (ratio, $1.04 \pm 0.003 ; N=21 ; p=$ $1.2 \times 10^{-7}$ by two-tailed $t$ test). As shown in Figure $1 B$, anti-Cplx4 fluorescence was negligible in PKC-positive synaptic terminals ratio, $1.07 \pm 0.02 ; N=22$ ) and was not significantly different from the ratio with secondary antibody only $(p=0.21$; two-tailed $t$ test). Therefore, within the sensitivity of immunocytochemistry, only Cplx3 was detectable in synaptic terminals of $\mathrm{ON}$, rod bipolar neurons.

\section{A peptide consisting of the SBD of Cplx3 increases spontaneous synaptic vesicle fusion}

Since one function of complexins at conventional synapses is to stabilize spontaneous release, we next asked whether ribbon-specific Cplx controls the rate of spontaneous vesicle fusion in mouse bipolar neurons in the absence of stimulation. To acutely interfere with Cplx function, we used a synthetic peptide consisting of the SNAREbinding region of mouse Cplx3 (Cplx-SBD; ATLRSHFRDKYRLPK; Vaithianathan et al., 2013), which is highly conserved in all four Cplx isoforms. As controls, we synthesized a peptide (Cplx-SBD/3A) in which three critical residues required for binding to the SNARE complex (Xue et al., 2007) were mutated (ATLASHFRDAARLPK), and a peptide consisting of the same residues as Cplx-SBD in scrambled order (Cplx-SBDscr; RSLYLRKPTADRFHK). Results using the two control peptides were indistinguishable and were combined. Peptides $(10 \mu \mathrm{M})$ were applied via dialysis in whole-cell recordings, with the patch pipette placed directly on the synaptic terminal, as shown in Figure $2 A$. In experiments with unlabeled Cplx peptides, fluorescent Ribeyebinding peptide (Zenisek et al., 2004) was included in the patch pipette to mark ribbons (Fig. $2 A$ ). In other experiments, Cplx peptides were labeled at the $\mathrm{N}$ terminus with 5-carboxyfluorescein to confirm the effectiveness of dialysis.

To monitor spontaneous neurotransmitter release, synaptic vesicles were stained by activity-dependent uptake of FM4-64 (Logiudice et al., 2009), as illustrated in Figure 2A. After break-in to begin whole-cell recording, cells were held under voltage clamp at $-65 \mathrm{mV}$ to prevent activation of voltage-gated calcium channels, and dye loss was measured as an index of spontaneous vesicle fusion. Under these conditions, FM-dye fluorescence in the synaptic terminal was relatively stable in the presence of control peptides (Fig. 2C), indicating that little spontaneous synaptic vesicle exocytosis occurred. However, in the presence of Cplx$\mathrm{SBD}$, fluorescence declined steadily after break-in (Fig. 2B,C), consistent with ongoing vesicle fusion despite the absence of calcium influx. Therefore, Cplx-SBD but not control peptides increased the rate of spontaneous release.

As an independent index of spontaneous synaptic vesicle fusion, we measured the change in membrane capacitance of the 
synaptic terminal in the presence of CplxSBD or control peptides, while holding the membrane potential at $-65 \mathrm{mV}$ under voltage clamp. In the presence of 10 $\mu \mathrm{M}$ Cplx-SBD, capacitance rose after break-in to an asymptotic increase of $217 \pm 20 \mathrm{fF}(N=11)$ within $\sim 40 \mathrm{~s}$ (Fig. $2 D)$. With control peptides, there was a significantly smaller increase of $66 \pm 32 \mathrm{fF}$ $(N=11 ; p=0.001$, two-tailed $t$ test $)$. Series conductance did not change significantly in either condition (Fig. 2E). The capacitance increase in the presence of Cplx-SBD is consistent with a higher rate of spontaneous synaptic vesicle exocytosis. Because FM4-64 fluorescence continued to decline steadily in the presence of Cplx-SBD (Fig. 2C), long after the capacitance increase had reached asymptote, the plateau of the capacitance shown in Figure $2 D$ likely reflects a balance between ongoing exocytosis and endocytosis, not cessation of vesicle fusion.

Increased spontaneous release could reflect elevated intracellular calcium in response to Cplx-SBD. However, basal fluorescence of the calcium indicator fluo-2 $(100 \mu \mathrm{M})$ was similar in the presence (931 \pm 215 arbitrary units; $N=6$ ) and absence of Cplx-SBD (756 \pm 131 arbitrary units; $N=7 ; p=0.49$; two-tailed $t$ test). Since Cplx-SBD does not elevate internal calcium, we suggest that it enhances spontaneous release by interfering with a clamping effect of native Cplx on calciumindependent vesicle fusion.

\section{Cplx3-SBD decreases release evoked by depolarization}

We next examined capacitance changes elicited by activation of calcium current under voltage clamp, to assess the effects of Cplx-SBD and control peptides on evoked fusion of synaptic vesicles. Examples of calcium current and changes in membrane capacitance $\left(C_{\mathrm{m}}\right)$ and series conductance $\left(G_{\mathrm{s}}\right)$ evoked by $500 \mathrm{~ms}$ depolarization are shown in Figure $3 A, B$ for control peptides and CplxSBD, respectively, and Figure $3 C$ summarizes the results. Although calcium current was not affected by Cplx-SBD, the inhibitory peptide significantly reduced the capacitance increase elicited by activation of calcium current (Fig. 3C). We therefore conclude that acute interference with Cplx3 decreases evoked release at mouse bipolar cell ribbon synapses, consistent with interference with a facilitatory action of Cplx 3 on stimulated exocytosis.

We also assessed evoked release in mouse bipolar cell terminals using FM4-64 destaining in terminals that were preloaded with the dye. To measure FM-dye loss, four to eight confocal images of the entire terminal were collected, followed by a $500 \mathrm{~ms}$ depolarization from -65 to $0 \mathrm{mV}$, and then another four to eight confocal images, as illustrated in Figure $3 D$ for control peptides and in Figure $3 E$ for Cplx-SBD. The drop in FM4-64 fluorescence in response to depolarization provided an index of the amount of

B
Control

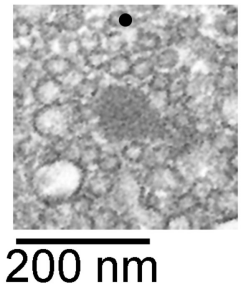

$\overline{200 \mathrm{~nm}}$

Figure 4. Cplx-SBD reduced reserve vesicles but not vesicles associated with synaptic ribbons in the terminals of mouse bipolar marizes the number of vesicles associated with synaptic ribbons in single electron micrographs in the presence of control peptide $(N=14)$ or Cplx-SBD $(N=11)$. Graph (right) shows the density of cytoplasmic reserve vesicles in the same set of electron micrographs. C, Examples of cytoplasmic vesicles not associated with ribbons.

vesicle fusion triggered by the stimulus. As shown in Figure $3 F$, the percentage decrease in FM4-64 fluorescence in the presence of Cplx-SBD was significantly less than the destaining in the presence of control peptides. This result is consistent with the reduced capacitance change observed with Cplx SBD, providing further indication that Cplx3 facilitates evoked exocytosis at mouse bipolar cell ribbon synapses.

\section{Electron microscopy of cytoplasmic reserve synaptic vesicles} and ribbon-associated synaptic vesicles

Decreased evoked release produced by Cplx-SBD could reflect a reduction in the number of synaptic vesicles associated with synaptic ribbons, which are thought to constitute the readily releasable pool (Matthews and Fuchs, 2010). To examine this possibility, mouse bipolar neurons were dialyzed via a whole-cell patch pipette with Cplx-SBD or control peptide while held at $-65 \mathrm{mV}$ without stimulation, and then fixed and examined by electron microscopy. Vesicles on ribbons were visualized using stereograms of single ribbons (Fig. $4 A$ ) to better resolve overlapping vesicles within a section. Similar numbers of vesicles were 
associated with ribbons in the presence of Cplx-SBD and control peptide (Fig. 4B), suggesting that reduced evoked release with Cplx-SBD reflects impaired exocytosis of vesicles in the releasable pool, rather than a smaller pool size. We also measured the density of synaptic vesicles in the cytoplasm, not associated with ribbons, and found that this reserve pool of vesicles was significantly reduced by Cplx-SBD compared with control peptide (Fig. $4 B, C)$. Decreased cytoplasmic vesicle density is consistent with the steady-state transfer of vesicle membrane to the plasma membrane caused by elevated spontaneous fusion in the presence of Cplx-SBD, which generated an increase in membrane capacitance (Fig. $2 D$ ) corresponding to $\sim 7000$ synaptic vesicles. We estimate from electron microscopy that the total vesicle population in the terminal falls by $\sim 5900$ with Cplx-SBD, approximately equivalent to the capacitance increase.

\section{Discussion}

The inhibitory peptide Cplx-SBD enhanced spontaneous release in mouse retinal bipolar neurons, measured both by destaining of FM4-64 and by increased steady-state membrane capacitance. A simple interpretation is that Cplx-SBD interferes with a stabilizing influence of native Cplx on the pool of releasable vesicles in the absence of stimulation, as suggested previously in studies of zebrafish bipolar neurons and photoreceptor cells, based on destabilization of spontaneous release by peptide interference and antisense morpholino knockdown of Cplx (Vaithianathan et al., 2013). Because Cplx 3 is the principal subtype detected in mouse bipolar neurons (Fig. 1), Cplx3 may act at bipolar cell ribbon synapses to arrest partially zippered SNARE complexes and prevent full fusion in the absence of stimulation, in the manner suggested for Cplx1 and Cplx2 at conventional synapses (Brose, 2008; Maximov et al., 2009; Xue et al., 2010; Yang et al., 2010; Hobson et al., 2011; Martin et al., 2011).

The suggested stabilizing action of Cplx 3 on spontaneous release at ribbon synapses is at odds with previous work in which Cplx3 was unable to restore the normal rate of spontaneous release at conventional synapses of CNS neurons after knockdown of Cplx1 (Kaeser-Woo et al., 2012), a failure that was attributed to differences in C-terminal domains of Cplx1 and Cplx3 (Yang et al., 2013). One explanation of the discrepancy is that Cplx function varies across cell types and species, a point emphasized by Yang et al. (2013). Therefore, Cplx3 might behave differently in its normal context at ribbon synapses, perhaps because of molecular differences between conventional and ribbon synapses, such as the presence of different syntaxin subtypes (Curtis et al., 2008, 2010). Another possibility is that Cplx 3 does not in fact affect spontaneous release at ribbon synapses, and binding of CplxSBD to SNARE complexes directly induces spontaneous exocytosis in some manner that does not involve competition with native Cplx3. The latter possibility could in principle be tested in future work by applying Cplx-SBD in Cplx3-knock-out cells to determine whether it is still capable of increasing spontaneous release.

In addition to increasing spontaneous release, Cplx-SBD also reduced evoked synaptic vesicle fusion in terminals of mouse bipolar neurons, measured both by FM4-64 destaining and by capacitance responses. In this regard, the action of Cplx-SBD differs from its effect in zebrafish bipolar neurons, where CplxSBD did not affect evoked release (Vaithianathan et al., 2013). The reason for this species' difference is unclear, but one possibility is that multiple Cplx $3 / 4$ subtypes might be expressed in zebrafish bipolar neurons (Zanazzi and Matthews, 2010), one or more of which might be less sensitive to interference by Cplx-
SBD. The effect of Cplx-SBD on evoked release shown here suggests that $\mathrm{Cplx} 3$ acts on evoked release at mammalian ribbon synapses much like Cplx1/2 at conventional synapses. That is, binding of Cplx3 to the SNARE complex facilitates subsequent fusion in response to calcium influx. A dual effect of Cplx3 on both spontaneous and evoked release at mouse ribbon synapses is similar to the dual roles reported for complexin at invertebrate synapses (Hobson et al., 2011; Martin et al., 2011), which underscores the conservation of complexin function across species, even for an isoform, Cplx3, that has been proposed not to stabilize spontaneous release (Kaeser-Woo et al., 2012; Yang et al., 2013).

Electron microscopy showed $\sim 20 \%$ loss of reserve synaptic vesicles in the presence of Cplx-SBD, consistent with elevated spontaneous release. Despite this reduction in reserve pools, the number of vesicles attached to ribbons was unaffected by Cplx$\mathrm{SBD}$, which suggests that the remaining reserve vesicles were sufficient to fully populate ribbons. Since the vesicles attached to ribbons are thought to represent the readily releasable pool (Matthews and Fuchs, 2010), depletion of the reserve pool is unlikely to account for the reduction in evoked release in the presence of Cplx-SBD. We suggest instead that the effect of Cplx-SBD on evoked exocytosis stems from interference with a facilitatory effect of native $\mathrm{Cplx} 3$ on calcium-triggered fusion.

Previous work on Cplx3 and Cplx4 double knock-out (DKO) mice has shown that the b-wave of the electroretinogram is reduced (Reim et al., 2009), suggesting impaired synaptic transmission from photoreceptor cells to ON bipolar neurons. In addition, On and Off responses of retinal ganglion cells are differentially decreased in DKO mice in an intensity-dependent manner (Landgraf et al., 2012), which was interpreted to indicate little functional effect at ON bipolar cell synapses in DKO animals. However, loss of Cplx3 and Cplx4 affects multiple synaptic sites in retinal circuitry, including photoreceptors, bipolar neurons, and AII amacrine cells (Landgraf et al., 2012), making it difficult to assign a precise synaptic function for these Cplx subtypes based on overall circuit behavior. An additional complication is that our results predict that spontaneous release would be elevated at synapses of photoreceptors and bipolar cells in DKO retina, providing an illumination-independent signal that mimics darkness in the case of photoreceptors and OFF bipolar cells and light in the case of ON bipolar cells. Therefore, reduced preparations, like ours, that allow direct measurements of synaptic vesicle fusion at a single synapse are more straightforward for determining the physiological roles of ribbon-specific complexin subtypes.

\section{References}

Brose N (2008) For better or for worse: complexins regulate SNARE function and vesicle fusion. Traffic 9:1403-1413. CrossRef Medline

Curtis LB, Doneske B, Liu X, Thaller C, McNew JA, Janz R (2008) Syntaxin $3 \mathrm{~b}$ is a t-SNARE specific for ribbon synapses of the retina. J Comp Neurol 510:550-559. CrossRef Medline

Curtis L, Datta P, Liu X, Bogdanova N, Heidelberger R, Janz R (2010) Syntaxin $3 \mathrm{~B}$ is essential for the exocytosis of synaptic vesicles in ribbon synapses of the retina. Neuroscience 166:832-841. CrossRef Medline

Hobson RJ, Liu Q, Watanabe S, Jorgensen EM (2011) Complexin maintains vesicles in the primed state in C. elegans. Curr Biol 21:106-113. CrossRef Medline

Kaeser-Woo YJ, Yang X, Südhof TC (2012) C-terminal complexin sequence is selectively required for clamping and priming but not for $\mathrm{Ca}^{2+}$ triggering of synaptic exocytosis. J Neurosci 32:2877-2885. CrossRef Medline

Landgraf I, Mühlhans J, Dedek K, Reim K, Brandstätter JH, Ammermüller J (2012) The absence of Complexin 3 and Complexin 4 differentially im- 
pacts the ON and OFF pathways in mouse retina. Eur J Neurosci 36:24702481. CrossRef Medline

LoGiudice L, Sterling P, Matthews G (2008) Mobility and turnover of vesicles at the synaptic ribbon. J Neurosci 28:3150-3158. CrossRef Medline

Logiudice L, Sterling P, Matthews G (2009) Vesicle recycling at ribbon synapses in the finely branched axon terminals of mouse retinal bipolar neurons. Neuroscience 164:1546-1556. CrossRef Medline

Martin JA, Hu Z, Fenz KM, Fernandez J, Dittman JS (2011) Complexin has opposite effects on two modes of synaptic vesicle fusion. Curr Biol 21:97105. CrossRef Medline

Matthews G, Fuchs P (2010) The diverse roles of ribbon synapses in sensory neurotransmission. Nat Rev Neurosci 11:812-822. CrossRef Medline

Maximov A, Tang J, Yang X, Pang ZP, Südhof TC (2009) Complexin controls the force transfer from SNARE complexes to membranes in fusion. Science 323:516-521. CrossRef Medline

Neher E (2010) Complexin: does it deserve its name? Neuron 68:803-806. CrossRef Medline

Reim K, Wegmeyer H, Brandstätter JH, Xue M, Rosenmund C, Dresbach T, Hofmann K, Brose N (2005) Structurally and functionally unique complexins at retinal ribbon synapses. J Cell Biol 169:669-680. CrossRef Medline

Reim K, Regus-Leidig H, Ammermüller J, El-Kordi A, Radyushkin K, Ehrenreich $\mathrm{H}$, Brandstätter JH, Brose N (2009) Aberrant function and structure of retinal ribbon synapses in the absence of complexin 3 and complexin 4. J Cell Sci 122:1352-1361. CrossRef Medline

Schmitz F (2009) The making of synaptic ribbons: how they are built and what they do. Neuroscientist 15:611-624. CrossRef Medline

Vaithianathan T, Zanazzi G, Henry D, Akmentin W, Matthews G (2013)
Stabilization of spontaneous neurotransmitter release at ribbon synapses by ribbon-specific subtypes of complexin. J Neurosci 33:8216-8226. CrossRef Medline

Van Wart A, Trimmer JS, Matthews G (2007) Polarized distribution of ion channels within microdomains of the axon initial segment. J Comp Neurol 500:339-352. CrossRef Medline

Xue M, Reim K, Chen X, Chao HT, Deng H, Rizo J, Brose N, Rosenmund C (2007) Distinct domains of Complexin I differentially regulate neurotransmitter release. Nat Struct Mol Biol 14:949-958. CrossRef Medline

Xue M, Craig TK, Xu J, Chao HT, Rizo J, Rosenmund C (2010) Binding of the complexin $\mathrm{N}$ terminus to the SNARE complex potentiates synapticvesicle fusogenicity. Nat Struct Mol Biol 17:568-575. CrossRef Medline

Yang X, Kaeser-Woo YJ, Pang ZP, Xu W, Südhof TC (2010) Complexin clamps asynchronous release by blocking a secondary $\mathrm{Ca}^{2+}$ sensor via its accessory $\alpha$ helix. Neuron 68:907-920. CrossRef Medline

Yang X, Cao P, Südhof TC (2013) Deconstructing complexin function in activating and clamping $\mathrm{Ca}^{2+}$-triggered exocytosis by comparing knockout and knockdown phenotypes. Proc Natl Acad Sci U S A 110:2077720782. CrossRef Medline

Zanazzi G, Matthews G (2009) The molecular architecture of ribbon presynaptic terminals. Mol Neurobiol 39:130-148. CrossRef Medline

Zanazzi G, Matthews G (2010) Enrichment and differential targeting of complexins 3 and 4 in ribbon-containing sensory neurons during zebrafish development. Neural Dev 5:24. CrossRef Medline

Zenisek D, Horst NK, Merrifield C, Sterling P, Matthews G (2004) Visualizing synaptic ribbons in the living cell. J Neurosci 24:9752-9759. CrossRef Medline 